[2] Tao S, C, Yuan T, Zhang Y. L,et al.Exosomes derived from miR-140-5poverexpressing human synovial mesenchymal stem cells enhance cartilage tissue regeneration and prevent osteoarthritis of the knee in a rat model. Theranostics, 2017. 7(1): p. 180-195.

[3] Chen L, et al. BMSCs-derived miR-223-containing exosomes contribute to liver protection in experimental autoimmune hepatitis. Mol Immunol, 2018. 93: p. 38-46. DOI:10.1016/j.molimm.2017.11.008.

[4] Wen D, Peng Y, Liu D, et al.Mesenchymal stem cell and derived exosome as small RNA carrier and Immunomodulator to improve islet transplantation. J Control Release, 2016. 238: p. 166-175.DOI:10.1016/j. jconrel.2016.07.044

Acknowledgement: I confirm that there is no conflict of interest

Disclosure of Interests: $\mathrm{Na}$ Zhang: None declared, Liyun Zhang: None declared, Gailian Zhang: None declared, Jin-Fang Gao Employee of: Shanxi Dayi Hospital Affiliated to Shanxi Medical University, Dan Ma: None declared, Juan Li: None declared, Ke Xu: None declared

DOI: 10.1136/annrheumdis-2019-eular.6452

\section{AB0148 OSTEOBLASTS SECRETE A HIGH LEVEL OF OSTEOPROTEGERINE AND SUPPRESS OSTEOCLASTOGENESIS BY INHIBITING SOLUBLE RANKL - IMPLICATIONS FOR THE PATHOGENESIS OF RHEUMATOID ARTHRITIS}

Hiroaki Yazawa, Yoshimi Aizaki, Yasuto Araki, Kojiro Sato, Toshihide Mimura Saitama Medical University, Department of Rheumatology and Applied Immunology, Saitama, Japan

Background: Osteoclasts (OCs) are multinucleated cells of monocyte/macrophage lineage and are the only cells known to resorb bone matrix. Osteoblasts (OBs) have the ability to differentiate bone marrow cells (BMCs) into osteoclasts when co-cultured with BMCs in the presence of Vitamin $D_{3}\left(1,25(O H)_{2} D_{3}\right)$ and prostaglandin $E_{2}\left(P E_{2}\right)$. OBs produce both $\mathrm{M}-\mathrm{CSF}$, a survival factor for OC precursor cells, and RANKL, a differentiation factor. In rheumatoid arthritis (RA), synoviocytes may fulfill the functional role of OBs, since OBs are not observed in the synovium of the joints. Although human BMCs are difficult to obtain, mouse BMCs are known to respond to human M-CSF and RANKL, and differentiate into OCs in the presence of both cytokines.

Objectives: Our aim was to examine whether a co-culture system of human synoviocytes and mouse BMCs might be developed. Through this analysis, we attempted to clarify the characteristics of synoviocytes in the pathogenesis of bone destruction observed in RA

Methods: Human synoviocytes were prepared from synovium obtained in the course of joint replacement surgical operations performed at Saitama Medical University Hospital. Written informed consent, approved by the ethics committee of the hospital, was obtained from each patient prior to the experiment. Mouse OBs were obtained from the calvaria of newborn C57BL/6 mice. Mouse BMCs were co-cultured with either synoviocytes or OBs. OCs were detected by tartrateresistant acid phosphatase (TRAP) staining. The concentrations of M-CSF, RANKL and the decoy receptor for RANKL, OPG, were quantified by ELISA in the culture supernatant of synoviocytes or OBs.

Results: When synoviocytes and BMCs were cultured with $1,25(\mathrm{OH})_{2} \mathrm{D}_{3}$ and $\mathrm{PGE}_{2}$, OCs were not induced. OC precursor cells did not seem to even survive. Next, we added human M-CSF to the system. This time, OC precursor cells remained, but TRAP-positive multinuclear cells were not observed. It was only in the presence of exogenous M-CSF and RANKL that BMCs transformed into OCs. RANKL was not detected in the culture supernatant of synoviocyte culture, but OPG and M-CSF were detectable at a level comparable to mouse OBs. Finally, we cultured mouse OBs and BMCs that were separated by a polycarbonate membrane in the presence of $1,25(\mathrm{OH})_{2} \mathrm{D}_{3}$ and $P G E_{2}$. In this case, too, BMCs did not survive unless exogenous M-CSF was added, and they did not differentiate into OCs unless both M-CSF and RANKL were added to the system.

Conclusion: These results indicate that the M-CSF derived from OBs or synoviocytes is not sufficient to support the survival of OC precursors. Likewise, the soluble RANKL derived from OBs is by itself insufficient for the differentiation of OCs, confirming the importance of a cell-cell interaction between $\mathrm{OBs}$ and $\mathrm{OC}$ precursors. Thus, membrane-bound RANKL seems to play a role more important than soluble RANKL in the co-culture system of OCs in vitro. Soluble RANKL may be neutralized by the OPG that is produced at a fairly high level from both OBs and synoviocytes. We propose that this is the system by which ectopic differentiation of OCs is prevented.

Disclosure of Interests: None declared

DOI: 10.1136/annrheumdis-2019-eular.6410

\section{$\mathrm{AB} 0149$ \\ ARSENIC TRIOXIDE IMPROVES TREG AND TH17 BALANCE VIA MODULATING STAT3 IN TREATMENT- NAÏVE RA PATIENTS}

Yue Zhang ${ }^{1,2}$, LI Chunling ${ }^{1}$, Zhiyi Zhang ${ }^{1}$, on behalf of Precision medicine center for Rheumatic diseases, First clinical college of Harbin Medical University. ${ }^{1}$ First Clinical College of Harbin Medical University, Rheumatology and Immunology, Harbin, China; ${ }^{2}$ Shenzhen Futian Hospital for Rheumatic Diseases, Shenzhen, China

Background: We have a long-term interest in novel arsenic trioxide $\mathrm{As}_{2} \mathrm{O}_{3}$ (ATO) medical application since some acute promyelocytic leukemia (APL) patients have been cured by first using ATO and survived for more than 45 years success fully treated by Prof. Zhang Tingtong in our institute. Moreover, our previous studies have shown that ATO significantly suppress angiogenesis and induced fibroblast like synoviocytes (FLS) apoptosis in collagen induced arthritis (CIA) model[1, 2]. However, the extract mechanism by which ATO anti-rheumatic and whether this occurs via modulation of immune system are still unclear.

Objectives: To investigated the immunologic mechanism by which ATO may inhibit $\mathrm{T}$ helper 17 cells (Th17) differentiation while promote regulatory $\mathrm{T}$ cells (Treg) generation via modulating signal transducer and activator transcription 3 (STAT3) in treatment-naïve RA patients.

Methods: Naïve $\mathrm{CD}^{+} \mathrm{T}$ cells sorted by fluorescence-activated cell sorting (FACS) from treatment-naïve RA patients and healthy controls were used to investigated the effect of ATO on its polarization process and related cytokines. Knockown or enforced expression of STAT3 transfection experiments were conducted by small interfering RNA (siRNA) and lentivirus STAT3 to verify the mecha nism of ATO on Th17/Treg balance in vitro. Collagen-induced arthritis (CIA) model was constructed to detect the clinical score, histopathological change bone destruction, Th17/Treg proportion and joint tissues immunohistochemistry. Single cell sequencing and other methods have been appplied too.

Results: We found that ATO prevented activated naïve CD4 ${ }^{+} T$-cell differentiate into Th17 cell and reduced cytokine production by activated Th17 cells by downregulating their signature transcription factors, STAT3 and orphan nuclear receptor (RORyt). Notably, ATO reduced Th17 cells frequency while increased Treg cells frequency under specific polarizing conditions from treatment-naïve RA patients by transfecting siRNA STAT3 and lentivirus STAT3. Furthermore, we have noticed that intervention of ATO in CIA model attenuated joint inflammatory infiltration and bone destruction, significantly improved the imbalanced Treg/Th17 ratio. In-detail single cell sequeining analysis is ongoing.

Conclusion: ATO may be a immune modulator candidate for treatment-naïve RA patients via balancing well Treg/Th17 cell ratio through STAT3 regulation.

\section{REFERENCES}

[1] Zhang, J., et al., Inhibition of angiogenesis by arsenic trioxide via TSP-1TGF-beta1-CTGF-VEGF functional module in rheumatoid arthritis. Oncotarget, 2017. 8(43): p. 73529-73546.

[2] Mei, Y., et al., Arsenic trioxide induces apoptosis of fibroblast-like synoviocytes and represents antiarthritis effect in experimental model of rheumatoid arthritis. J Rheumatol, 2011. 38(1): p. 36-43.

[3] Vasheghani, F., et al., PPARgamma deficiency results in severe, accelerated osteoarthritis associated with aberrant mTOR signalling in the articular cartilage. Ann Rheum Dis, 2015. 74(3): p. 569-78.

Acknowledgement: National Science Foundation of China (NO. 81273291) and (NO. 81771748,81771749)

Disclosure of Interests: None declared

DOI: 10.1136/annrheumdis-2019-eular.8330

\section{AB0116 BROMODOMAIN INHIBITOR, I-BET762 INHIBITS PRODUCTION OF PRO-INFLAMMATORY MEDIATORS BY DOWN-REGULATING BROMODOMAIN AS AN EPIGENETIC READER IN RHEUMATOID ARTHRITIS FIBROBLAST-LIKE SYNOVIOCYTES}

Ra Ham Kim ${ }^{1}$, Hyun Jung Yoo ${ }^{1,2}$, Shin Eui Kang ${ }^{1}$, Ji Soo Park ${ }^{1}$, Seon Uk Kim¹, Eun Young Lee 2 , Jin Kyun Park ${ }^{2}$, Yeong Wook Song ${ }^{1,2} .{ }^{1}$ Seoul National University, Department of Molecular Medicine and Biopharmaceutical Sciences, Graduate School of Convergence Science and Technology, and College of Medicine, Seoul, Korea, Rep. of (South Korea); ${ }^{2}$ Seoul National University Hospital, Division of Rheumatology, Department of Internal Medicine, Seoul, Korea, Rep. of (South Korea)

Background: Rheumatoid arthritis (RA) is a chronic autoimmune disorder, characterized by joint inflammation and bone destruction. The fibroblast-like synoviocyte contributes to the pathogenesis of RA through proliferation and production of cytokines. Recently, blockade of the bromodomain and extra-terminal domain 
(BET) family protein was reported to be a potential therapeutic target by inhibiting epigenetics interaction between bromodomains and acetylated histones.

Objectives: We aimed to investigate the effects of bromodomain-containing protein 4 (BRD4) inhibition on tumor necrosis factor (TNF)- $\alpha$-stimulated rheumatoid arthritis fibroblast-like synoviocyte (RA-FLS) using BRD4 inhibitor, I-BET762. Methods: We examined the effects of BET protein inhibitor on cytokine production and cellular proliferation in RA-FLS. RA-FLSs were treated with TNF- $\alpha$ (10 $\mathrm{ng} / \mathrm{ml}$ ) in the presence of I-BET762 for $48 \mathrm{~h}$. The cell viability and proliferation were measured after 24, 48, and 72 h in I-BET762-treated RA-FLS using CCK-8 colorimetric assay. We then screened pro-inflammatory mediators by using the human inflammation antibody array (Abcam). The levels of interleukin (IL)-6 and IL-8 in culture supernatants of TNF- $\alpha$-stimulated RA-FLS were measured by enzyme-linked immunosorbent assays (ELISA).

Results: I-BET762 had no significant effect on cytotoxicity and proliferation of RA-FLS. I-BET762 reduced the secretion of CXCL-10 and regulated upon activation, normal T cell expressed, and secreted (RANTES) from RA-FLS while increased the secretion of tissue inhibitor of metalloproteinase 2 (TIMP-2) in the antibody array. In addition, ELISA showed significantly decreased level of IL-6 and IL-8 in culture supernatant after treatment with I-BET762.

Conclusion: Inhibition of BRD4 by I-BET762 leads to down-regulation of proinflammatory cytokines and chemokines in RA-FLS. I-BET762 may have antiinflammatory properties and provide a therapeutic target molecule in RA

\section{REFERENCES}

[1] Sahai V, et al. Oncotarget 2016;6;7(33):53997-54009.

[2] Klein K, et al. Ann Rheum Dis 2016;75: 422-429.

Disclosure of Interests: None declared

DOI: 10.1136/annrheumdis-2019-eular.6549

\section{AB0139 ASSOCIATION OF RS17004921 ADORA2A GENE POLYMORPHISM WITH EFFICACY OF METHOTREXATE IN PATIENTS WITH RHEUMATOID ARTHRITIS}

Vera Milic ${ }^{1}$, Milka Grk ${ }^{2}$, Nemanja Damjanov ${ }^{3}$, Biljana Jekic ${ }^{2} .{ }^{1}$ Institute of Rheumatology, Faculty of Medicine,Belgrade, Belgrade, Serbia; ${ }^{2}$ Institute of Human Genetics, Faculty of Medicine, Belgrade, Belgrade, Serbia; ${ }^{3}$ Institute of Rheumatology, Faculty of Medicine, Belgrade, Belgrade, Serbia

Background: Adenosine A2A receptors (ADORA2A) are part of the adenosinemediated antiinflammatory pathway. These receptors are over-expressed in peripheral blood leukocytes of patients with rheumatoid artritis (RA). Methotrexate (MTX), the gold standard for therapy, exerts its antiinflamatory effects via increased release of adenosine into the extracellular space. Adenosine binds to ADOR A2A and A3 and initiates an antiinflammatory response. Therefore, ADOR gene polymorphisms could have an impact on MTX therapy outcome.

Objectives: To examine the role of adenosine receptor $2 \mathrm{~A}$ gene ( $A D O R A 2 A$, rs 17004921) polymorhism on outcome of MTX treatment in RA.

Methods: A total of 123 patients with RA (mean age 56.5 \pm 10.73 , 91 (74\% female), treated with MTX for 6 months (mean dose 12.22 $\pm 3.20 /$ weekly) were enrolled in a prospective study. Genotypisation within ADORA2A gene was performed using the KASP genotyping assays. MTX efficacy assessment was based on the changes in the Disease activity score (DAS28) after 6 months of treatment according to the EULAR response criteria. Patients with good and moderate response were classified as „responders“, whereas patients with poor response were considered „nonresponders". Data of adverse effects were collected during this period. MTX efficacy and toxicity were compared among patients with different genotypes. All statiscal analyses were performed by SPSS version 16.0.

Results: Median DAS 28 at the beginning of MTX treatment was $7.43 \pm 0.89$. According to EULAR response criteria, 111 (90.2\%) patients with RA were classified as responders. Among all RA patients, 97 (78.9\%) had CC, 20 (16.3\%) CT and $6(4.9 \%)$ TT genotype. The distribution of ADORA2 genotypes in responders (CC $77.5 \%$, CT $17 \%$ and TT $5.4 \%$ ) was not significantly different from nonresponders (CC $91.7 \%$, CT 8.3\%), p>0.05. After 6 months, 26 carriers of T allele (CT +TT) had higher reduction DAS 28 (7.16 vs. 3.88) in comparison to other 97 patients (7.50 vs.4.64, $p=0.013)$. Adverse effects were reported in 24 (19.5\%) patients. Most of patients had hepatotoxicity and nausea, $14(58 \%)$ and $9(37 \%)$ respectively. No statistically significant association between ADORA2A genotype and side events has been observed $(p>0.05)$.

Conclusion: According to our results, T allele of ADORA2A rs17004921 polymorphism may have favourable influence on efficacy in RA patients treated with methotexatate.

\section{REFERENCES}

[1] Cronstein B, Sitkovsky M. Adenosine and adenosine receptors in the pathogenesis and treatment of rheumatic diseasess. www.nature.com/ nrrheum
[2] Hider SL, Thomson W, Mack LF, et al. Polymorhisms within the adenosine receptor $2 \mathrm{a}$ gene are associated with adverse events in RA patients treated with MTX. Rheumatology 2008;47:1156-1159.

Disclosure of Interests: Vera Milic: None declared, Milka Grk: None declared, Nemanja Damjanov Grant/research support from: AbbVie, Pfizer and Roche Consultant for: Abbvie, Gedeon Richter, Merck, Novartis, Pfizer and Roche. Speakers bureau: Abbvie, Gedeon Richter, Merck, Novartis, Pfizer and Roche., Biljana Jekic: None declared

DOI: 10.1136/annrheumdis-2019-eular.7830

\section{Spondyloarthritis - etiology, pathogenesis and animal models}

\section{AB0150 IMMATURE GRANULOCYTES LEVEL IS A POTENTIAL BIOMARKER IN PERIPHERAL ENTHESITIS}

Atheer Al-Ansari ${ }^{1}$, Iman Hussein ${ }^{2} .{ }^{1}$ Mediclinic Airport Road Hospital, Rheumatoloy, Abu Dhabi, United Arab Emirates; ' ${ }^{2}$ ifeline Hospital, Rheumatoloy, Abu Dhabi, United Arab Emirates

Background: Immature granulocytes (IG) level in peripheral blood is used as early sign of infection. On the other hand, IG could be elevated in other conditions like inflammatory or cancerous diseases and in pregnancy. Similar to CRP, we have observed a series of cases of peripheral enthesitis associated increased level of IG and correlated with CRP elevation and clinical activity.

Objectives: To test the concept of IG as a biomarker in peripheral enthesitis, This observation might be a reflection to the innate immunity role in pathogenesis of enthesitis.

Methods: We have identified 13 cases over the last 12 months at the Rheumatology clinic in our centre who have shared two features (clinical enthesitis and elevated IG) those 13 cases are mainly presented with recurrent foot and ankle pain and swelling of enthesitis nature.

Results: The cohort is of equal gender distribution $46 / 54 \%$ respectively and age range 31-53 years (mean age 38.5), only 3 patients are known to have Psoriatic arthritis whilst the rest either undiagnosed or diagnosed Rheumatoid arthritis or gout prior to clinic visit. The rheumatologist clinical diagnoses are of enthesitis of foot, ankle, knee and hip area as shown on the table [1] apart from one patient who has spinal symptoms mainly. 4/13 patients have the history of psoriasis and after their visits we find them fit in the CASPAR criteria for psoriatic arthritis. Al these cases are associated with increased absolute number of IG as well as differential (IG) number compare to $10 / 13$ of these cases are having high CRP. The 3 cases with normal CRP do have relatively slight increase in (IG) number. The main correlation is of response to therapy and was seen in 7 cases who have followed up at the time of submission and it shows a $100 \%$ correlation between CRP, absolute (IG) and differential (IG) values. There are 3 patients who have elevated (IG) but normal CRP and it would possible indicate (IG) test has a better yield than CRP in peripheral enthesitis.

Conclusion: Immature granulocytes can be elevated in inflammatory disease and more notably in peripheral enthesitis of the lower limbs of inflammatory rathe than mechanical nature and do correlate with CRP elevation as well as response to therapy. Larger studies are needed to assess the usefulness and validity of (IG) level in clinical practice.

\section{REFERENCES}

[1] Rudwaleit M et al, The Assessment of SpondyloArthritis International Society classification criteria for peripheral spondyloarthritis and for spondyloarthritis in genera, Ann Rheum Dis. 2011;70(1):25

[2] Moots $\mathrm{R}$ et al, Low-density granulocytes: functionally distinct, immature neutrophils in rheumatoid arthritis with altered properties and defective TNF signaling, Translational \& Clinical Immunology 2016 https://doi.org/ 10.1189/jlb.5A0116-022R

[3] Salvarani c et al, Isolated peripheral enthesitis and/or dactylitis: a subset of psoriatic arthritis. J Rheumatol. 1997 Jun;24(6):1106-10

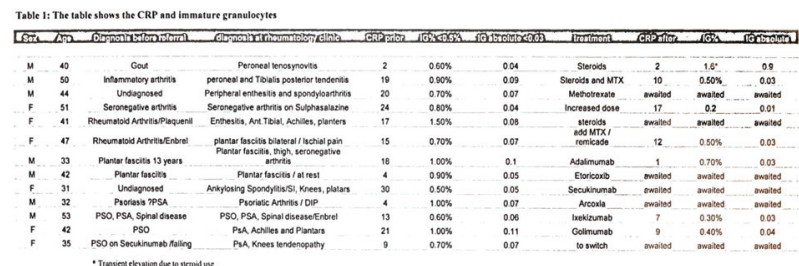

Disclosure of Interests: None declared DOI: 10.1136/annrheumdis-2019-eular.4348 